\title{
Optimal Scheduling of Logistical Support for Medical Resources Order and Shipment in Community Health Service Centers
}

\author{
Ming Liu, Liping Zhang, Zhe Zhang \\ School of Economics and Management, Nanjing University of Science and Technology, China \\ liumino@njust.edu.cn, pelovely@126.com, zhangzhe@,njust.edu.cn
}

Received: April 2015

Accepted: October 2015

\section{Abstract:}

Purpose: Medical resources scheduling affects the medical institution's operation cost, customer satisfaction and medical service quality. Therefore, a lean arrangement of medical resources order and shipment is quite necessary and important. In this paper, we propose two optimal models for medical resources order and shipment in community health service centers (CHSCs), with a dual emphasis on minimizing the total operation cost and improving the operation level in practice.

Design/methodology/approach: The first planning model is a deterministic planning model (DM). Systematically, it considers constraints including the lead time of the suppliers, the storage capacity of the medical institutions, and the integrated shipment planning in the dimensions of time and space. The problem is a multi-commodities flow problem and is formulated as a mixed 0-1 integer programming model. Considering the stochastic demand, the second model is constructed as a stochastic programming model (SM). A solution procedure is developed to solve the two models and a simulation-based evaluation method is presented to compare the performances of the proposed models.

Findings: The main contributions of this paper include the following two aspects: (1) most research on medical resources allocation studies a static problem taking no consideration of the time evolution and the time-varying demand. In this paper, time-space network technique is adopted to depict the logistics situation in CHSCs from both time and space dimensions. (2) 
the logistics plans in response to the deterministic demand and the time-varying demand are constructed as a 0-1 mixed integer programming model and a stochastic integer programming model, respectively. The optimal solutions can not only minimize the total operation cost, but also improve the order and shipment operation in practice.

Originality/value: Generally, medical resources in CHSCs are purchased by telephone or email. The important parameters in decision making, i.e., order/shipment frequency and order quantity, are manually determined by the decision maker based upon his/her experience. The planned schedules may not be efficient or feasible to satisfy all demands since a large portion of customer requests are uncertain and time-varying. The proposed methods in this paper could be effective in solving the problems in actual operations.

Keywords: time-space network; time-varying demand; scheduling; medical resources

\section{Introduction}

Last year, we conducted a research on medicine supply chain situation in CHSCs in Nanjing, China, by using the questionnaire survey method. The result shows that most CHSCs currently in this city do not use any electronic purchase systems or decision support systems to help optimize the ordering and scheduling work. Medical resources are always purchased by telephone or e-mail. The important parameters in decision making, i.e., order/shipment frequency and order quantity, are manually determined by the decision maker based on his/her experience. The planned schedules may not be efficient, or may not be feasible to satisfy all demands since a large portion of customer service requests in CHSCs are uncertain and time-varying ( $\mathrm{Li} \& \mathrm{Yu}, 2011$ ). The result of the questionnaire survey motivates us to improve the situation and to develop a systematic planning approach that takes all these factors into consideration.

In line with our survey, CHSC purchases medical resources from its upstream authorities, the District Center for Disease Control and Prevention (DCDC), and DCDC imports medical resources from the pharmaceutical companies (the suppliers). A lead time is required for the supplier, to produce the required medical resources. Similarly, a lead time is required for the DCDC to check the quality of medical resources. Generally, a compacted scheduling of medical resources order and shipment can not only efficiently reduce the operation cost, but also promote the medical service quality. However, to the best of our knowledge, although many studies have focused on medical resources scheduling, few of them consider the problem of medical resources scheduling problem in CHSCs with uncertain demands, lead time, as well as capacity constraint. 
In this paper, we consider the medical resources scheduling problem in CHSCs with time-varying demand, the lead time of supplier, the capacity constraint. Meanwhile, the scheduling problem integrates the shipment planning in the dimensions of time and space. The rest of this paper is organized as follows. Section 2 is the literature review. The modeling approach is introduced in Section 3. Section 4 presents the solution algorithm and the evaluation method. In Section 5 , numerical tests are performed. Finally, we conclude in Section 6.

\section{Literature Review}

Numerous studies have focused on medical resources scheduling, including medicine ordering, shipment and medical resources allocation. We briefly introduce them in the following paragraphs.

Initially, a most related empirical study is provided by Dib, Sun, Zhu, Wei and Li (2010). They investigated 58 community health centers and surveyed 372 residents randomly about their satisfaction towards these centers in Dalian, China. They suggested that the medicine supply chain for the community health centers should be improved and the superior departments support to the community health centers should be augmented.

In the second place, theory research with the topic of medical resources scheduling have been conducted by many experts. For example, Duintjer Tebbens, Pallansch, Alexander and Thompson (2010) proposed a mathematical framework for determining the optimal management of a vaccine stockpile over time. Sun, DePuy and Evans (2014) built mathematical models to optimize the patients' allocation considering two objectives related to patients' cost of access to healthcare services: (1) minimizing the total travel distance to hospitals; and (2) minimizing the maximum distance a patient travels to a hospital. Moreover, the models can help decision makers to predict a resources shortage during a pandemic influenza outbreak. Savachkin and Uribe (2012) presented a simulation optimization model to generate dynamic strategies for distribution of limited mitigation resources, such as vaccines and antivirals, over a network of regional outbreaks. The model can redistribute the resources remaining from previous allocations in response to changes in the pandemic progress. Vlah and Rui (2012) addressed the issue of scheduling medical treatments for resident patients in a hospital as a multi-objective binary integer programming (BIP) model and three types of heuristics were proposed and implemented to solve it. Rottkemper, Fischer and Blecken (2012) designed a mixed-integer programming model for distribution and inventory relocation under uncertainty in humanitarian operations. Rachaniotis, Dasaklis and Pappis (2012) presented a resources scheduling model in epidemic control with limited resources. The objective is to minimize the total amount of the infected people in a certain time horizon by relocating the available resources over several regions. Dasaklis, Pappis and Rachaniotis (2012) suggested 
several future research directions and defined the roles of logistics operations and their management may play in assisting the control of epidemic outbreaks.

Thirdly, as to the variability and uncertainty characteristics of the demand, Holte and Mannino (2013) presented that a major difficulty in medical resources allocation stems from the fact that such an allocation must be established several months in advance, and the exact number of patients for each specialty is an uncertain parameter. They modeled the uncertain problem as adjustable robust scheduling problem and developed a row and column generation algorithm to solve it. Beraldi, Bruni and Conforti (2004) considered the inherent uncertainty in emergency medical services and developed a stochastic programming model with probabilistic constraints, which aims to decide the location of the service sites and the amount of emergency vehicles to be assigned to each site. Zhang and Jiang (2014) presented a biobjective robust program to design a cost-responsiveness efficient emergency medical services (EMS) system under uncertainty. The proposed model simultaneously determined the location of EMS stations, the assignment of demand areas to EMS stations, and the number of EMS vehicles at each station to balance cost and responsiveness. Nikakhtar and Hsiang (2014) considered uncertain situations such as epidemic diseases that could affect the patient flow in a healthcare system by developing a discrete-event simulation model for a local community health clinic in Lubbock, Texas. To tackle the uncertain nature of emergency department and improve the resources management, $\mathrm{Xu}$, Wong and Chin (2014) used self-organizing map, kmeans, and hierarchical methods to group patients based on their medical procedures, and then discussed how the resulting patient groups can be used to enhance the emergency department resources planning.

In summary, the time-varying demand in CHSCs, with multiple medical resources types and the optimal scheduling of ordering and shipment are highly correlated with each other. It is difficult to use the traditional integer programming techniques to formulate and efficiently solve this type of problem. On the other hand, the time-space network method has been popularly employed to solve scheduling problems, which provides a natural and efficient way to represent multiple conveyance routings with multiple commodities in the dimensions of time and space. Although the resulting model scale is generally enlarged due to the extension in the dimension of time, complicated time-related constraints can normally be easily modeled for realistic problems, particularly in comparison with the space network models (Yan, Lin \& Lai, 2013). Coupled with the development of efficient algorithms, the time-space network models (usually formulated as multiple commodity network flow problems) can be effectively and efficiently solved (Kliewer, Mellouli \& Suhl, 2006; Yan, Lin \& Chen, 2014; Yan, Shih \& Shiao, 2008; Yan, Tang \& Fu, 2008). Therefore, time-space network technique could be suitable to solve the medical resources scheduling problem in CHSCs. 


\section{Modeling Approach}

In this section, we discuss the network structure and mathematical formulation for the planning of logistical support in CHSCs. A time-space network framework is employed to denote the medical resources order and shipment scheduling. Based on the time-space network, a deterministic planning model (DM) is developed to address the issue of knowing the demand in CHSCs in advance. A stochastic planning model (SM) is then presented to address the issue of stochastic demand in actual operations. In what follows, we will first introduce the time-space network that serves as the basis for our mathematical formulations.

\subsection{Network Structure}

The time-space network of logistical support in CHSCs denotes the potential order and shipment of the medical resources within a certain period and space locations, as shown in Figure 1. The vertical axis represents the supplier, the district center for disease control and prevention (DCDC) and the CHSCs, while the horizontal axis stands for the duration of time. Each node denotes the different department at a specific time. The shorter the time interval is, the more accurate the decision-making is. Three types of arcs are defined below.

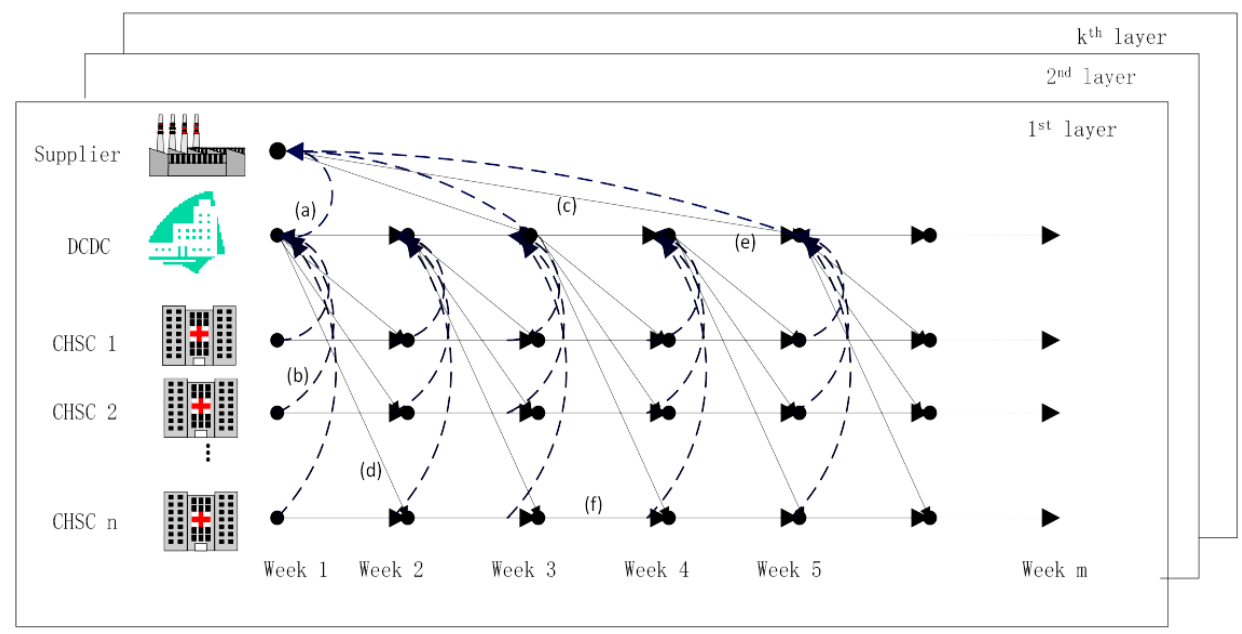

Figure 1. Time-space network of medical resources flows

(1) Ordering arc

An ordering arc (see (a-b) in Figure 1) represents an order from the DCDC to the supplier, or an order from the CHSC to the DCDC. While an ordering arc exists, an ordering cost is incurred no matter when the order takes place, and how many medical resources are purchased. Note that order operation is always completed by telephone or 
e-mail in practice, thus there is no physical flow on the ordering arc. The arc flow, which is a binary variable, denotes whether an order is placed or not. The arc flow's upper bound is one, indicating that an order takes place. Intuitively, the arc flow's low bound is zero.

(2) Shipment arc

A shipment arc (see [c-d] in Figure 1) represents medical resources are delivered from the supplier to the DCDC or from the DCDC to the CHSC. The cost for the shipment arc is also comprised of two parts, which are the constant cost that is incurred whenever the shipment takes place and regardless of the quantity of medical resources, and a variable cost represented by travel distance, carry hours used, meals etc, which is in proportion to the quantity of medical resources shipped. Since the shipment arc connects different depots, the arc flow's upper bound is the capacity of the DCDC or the CHSC, and the arc flow's low bound is zero.

(3) Holding arc

A holding arc (see [e-f] in Figure 1) represents the holding of medical resources at DCDC or CHSC. The arc cost denotes the inventory cost incurred by holding medical resources, which is in proportion to the stored quantity of medical resources on the arc. Therefore, the arc flow's upper bound is also the capacity of the node (DCDC or CHSC), and the arc flow's low bound is zero.

\subsection{The Deterministic Planning Model (DM)}

Before introducing the model's formulation, the notations and symbols are listed below:

\section{Sets}

$A^{k}$ : Set of all arcs in the $k^{\text {th }}$ layer of the time-space network.

$N^{k}$ : Set of all nodes in the $k^{\text {th }}$ layer of the time-space network.

$K$ : Set of the $k^{\text {th }}$ layer of the time-space network.

$H$ : Set of all holding arcs in the time-space network. 


\section{Parameters}

$c_{i j}^{k}$ : arc $(i, j)$ cost in the $k^{\text {th }}$ layer of the time-space network; if the arc is a ordering arc, the arc cost is the ordering cost; if the arc is a shipment arc, the arc cost is the shipment cost; if the arc is a holding arc, the arc cost is the inventory cost incurred by holding the medical resources.

$u_{i j}^{k}$ : arc $(i, j)$ flow's upper bound in the $k^{t h}$ layer of the time-space network.

$l_{i j}^{k}$ : arc $(i, j)$ flow's lower bound in the $k^{\text {th }}$ layer of the time-space network.

$u_{i j}$ : storage capacity (DCDC or CHSC)for the holding arc $(i, j)$ flow.

$a_{i}^{k}$ : the supply or demand of medical resources at node $i$ in the $k^{\text {th }}$ layer of the time-space network; if $a_{i}^{k} \geq 0$, the supply of medical resources; if $a_{i}^{k}<0$, the demand of medical resources; at the time slot for beginning dispatching, the supply at the DCDC and the CHSC equals to its storage capacity.

\section{Decision variables}

$x_{i j}^{k}: \operatorname{arc}(i, j)$ flow in the $k^{\text {th }}$ layer of the time-space network.

Based on the notations, the mathematical formulation of DM can be formulated as follows:

$$
\begin{gathered}
\text { Min: } Z=\sum_{k \in K} \sum_{i j \in A} c_{i j}^{k} x_{i j}^{k}, \\
\text { s.t. : } \sum_{j \in N^{k}} x_{i j}^{k}-\sum_{l \in N^{k}} x_{l i}^{k}=a_{i}^{k}, \quad \forall i \in N^{k}, k \in K, \\
\sum_{k \in K} x_{i j}^{k} \leq u m_{i j}, \quad \forall i j \in H, \\
l_{i j}^{k} \leq x_{i j}^{k} \leq u_{i j}^{k}, \quad \forall i j \in A^{k}, k \in K, \\
x_{i j}^{k} \in I, \quad \forall i j \in A^{k}, k \in K .
\end{gathered}
$$

The objective function (1) minimizes the sum of the operation cost, including the ordering cost, the shipment cost and the holding cost. Constraint (2) is the flow conservation constraint for each node in the time-space network. Constraint (3) is the capacity constraints. Constraint (4) guarantees that all arc flows are within their bounds. Constraint (5) ensures that all flow variables are integers. 
Since all constraints and cost functions in this optimization model are linear, the proposed multi-commodity flow problem is formulated as a mixed 0-1 integer programming model. The optimal result can be put to practical use if we can identify the demand at each node in the time-space network in advance. However, a large part of the demand for medical resources are stochastic and are difficult to be accurately forecasted, which make the planned medicine scheduling unable to satisfy all those demands that suddenly pop up. Therefore, we need to improve the model to make it more realistic and practical.

\subsection{The Stochastic Planning Model (SM)}

The network structure of the SM is the same to the network of the DM, except that the demand at each node in the time-space network is uncertain. It is worth mentioning that only the normal stochastic demand is considered in this work. Large-scale disruption of the demand which may be caused by some unexpected public health incidents (i.e., SARS) goes beyond our research scope. To formulate the SM, we set more notations and symbols as follows in addition to those already introduced.

\section{Set}

$\Omega$ : the set of stochastic situations.

\section{Parameters}

$a_{i}^{k}(\omega)$ : the stochastic supply or demand for medical resources at node $i$ in the $k^{\text {th }}$ layer of the time-space network; if $a_{i}^{k} \geq 0$, the stochastic supply of medical resources; if $a_{i}^{k}<0$, the stochastic demand of medical resources; at the time slot for beginning dispatching, the stochastic supply at the DCDC and the CHSC is still set to be its storage capacity.

$E(\omega)$ : Excepted cost of the logistics arcs with the stochastic demand.

\section{Decision variables}

$x_{i j}^{k}(\omega)$ : arc $(i, j)$ flow in the $k^{t h}$ layer of the time-space network with the stochastic situation $\omega$. 
Based on the notations, the SM can be formulated as follows:

$$
\begin{gathered}
\text { Min: } Z=E\left(\sum_{k \in K} \sum_{i j \in A} c_{i j}^{k} x_{i j}^{k}(\omega)\right), \\
\text { s.t. : } \sum_{j \in N^{k}} x_{i j}^{k}(\omega)-\sum_{l \in N^{k}} x_{l i}^{k}(\omega)=a_{i}^{k}(\omega), \quad \forall i \in N^{k}, k \in K, \omega \in \Omega, \\
\sum_{k \in K} x_{i j}^{k}(\omega) \leq u m_{i j}, \quad \forall i j \in H, \omega \in \Omega, \\
l_{i j}^{k} \leq x_{i j}^{k}(\omega) \leq u_{i j}^{k}, \quad \forall i j \in A^{k}, k \in K, \omega \in \Omega, \\
x_{i j}^{k}(\omega) \in I, \quad \forall i j \in A^{k}, k \in K, \omega \in \Omega .
\end{gathered}
$$

Similarly, the objective function (6) minimizes the excepted value of the operation cost. Constraint (7) is the flow conservation constraint for each node in the time-space network. Constraint (8) is the capacity constraint with stochastic demand. Constraint (9) guarantees that all arc flows with stochastic demand are within their bounds. Constraint (10) ensures that all flow variables with stochastic demand are integers. Since all decision variables are time-varying with the stochastic demand, the proposed problem can be processed as a stochastic integer programming model. The optimal result would be more realistic and practical.

\section{Solution Procedure and Evaluation Method}

In this section, we will discuss how to solve the proposed models and how to evaluate them based on a simulation method.

\subsection{Solution Procedure}

The DM is formulated as a mixed 0-1 integer programming model and it can be solved within a reasonable time, by using the mathematical tool MATLAB, coupled with the optimal software CPLEX 12.4. The SM is formulated to depict the stochastic demand at each time point, and the model is constructed as a stochastic integer programming model. Given the demand for each node in the time-space network, the SM can be solved as a deterministic planning model. Therefore, the solution procedure for the SM is described as follows: 


\section{Procedure for the SM:}

Input: Initial parameters in the SM and the distribution function of demand.

Output: The optimal scheduling and the operation cost of the medical resources order and shipment for the DCDCs and the CHSCs.

\section{Begin}

Initialization, set $C$ as the number of simulation times, $c=1,2, \ldots, n$;

$c \leftarrow 1$

while (not termination condition) do

1. Randomly generate the demand for each CHSC in the time-space network according to the distribution function;

2. Solve the mixed 0-1 integer programming model by using the MATLAB compiler, coupled with CPLEX 12.4;

3. Record the optimal schedules and the operation cost.

$c \leftarrow c+1$

end

4. Calculate the average operation cost as the final result.

5. Output optimal scheduling and the operation cost.

\section{End}

\subsection{Evaluation Method}

In practice, a classic order strategy, the $(t, S)$ strategy, has always been adopted to manage the medicine inventory in both CHSC and DCDC. That means, the CHSC and the DCDC will import medical resources with a fixed time interval. The purpose of the order is to keep the stock of medical resources at a certain level. Herein, we address it as the actual operations of medical resources scheduling and we abbreviate it as AOM. Similarly in the SM, demand for 
each node in the time-space network is randomly generated. The first difference between the AOM and the SM is the order quantity, which is equal to the capacity of the node minus the available quantity of medical resources when decision making. The second difference between these two models is the fixed time interval, which is set to be two weeks. Similarly, the AOM can be solved by using the above solution procedure.

The performances of the DM, the SM, and the AOM are evaluated via a simulation test. We first use the average demand of the historical demand data to complete the DM calculation. Next we randomly generate the stochastic demand data based on the average demand with a certain standard deviation, and input them into the SM and then solve it. After that, we fix the ordering time interval and adopt the $(t, S)$ strategy to complete the AOM calculation. Finally, we compare the DM, the SM and the AOM with statistical results.

\section{Numerical Tests}

To test how well the models may be applied in the real world, we performed numerical tests using operating data from 5 CHSCs in Nanjing, China, with reasonable simplifications. The tests were performed on a personal computer equipped with a Intel (R) Core (TM) $3.10 \mathrm{GHz}$ CPU and $4.0 \mathrm{~Gb}$ of RAM in the environment of Microsoft Windows 7.

\subsection{Parameters Setting}

This numerical example focuses on the scheduling of logistical support for medical resources order and shipment in CHSCs. The planning period is set to be half a year ( 26 weeks). Lead time of the supplier is set to be 2 weeks, and lead time of the DCDC is 1 week. Each layer of the time-space network, which represents a kind of the medicine, involves 1 supplier, 1 DCDC and 5 CHSCs. The historical data of the order quantity for each kind of vaccines in the past years, from January 2011 to June 2013, was collected when we conducted the questionnaire survey in the CHSCs in Nanjing, China. For example, the historical data of influenza vaccine during these years in a CHSC is shown in Figure 2. According to the historical data, we can calculate the average demand for each kind of vaccines at each week. The standard deviation of the stochastic demand is set to be 10 . In practice, the decision makers can adjust these parameters according to the actual situation. 


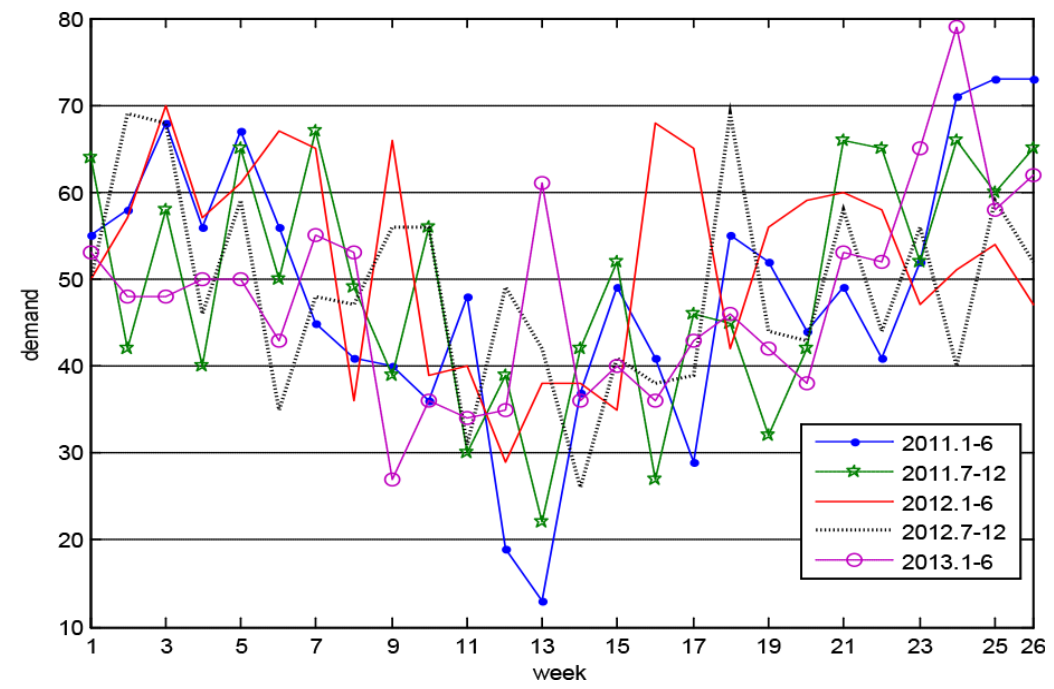

Figure 2. Historical data of influenza vaccine

\subsection{Test Results}

As introduced above, we use the AOM to simulate the actual operation of the medical resources order and shipment, and we present two other methods, the DM and the SM, to address with different demand situations. DM is designed to deal with the scheduling when demand at each time point is preset in advance, and SM is proposed to complete the planning when demand is uncertain. The performances of these three methods are shown in Table 1. The objective value of the SM (638087.2) is the smallest one, which is $35.4 \%$ lower than the operation cost of the AOM (987950.1). Similarly, the objective value of the DM is 643167.5, which is $34.8 \%$ lower than the cost of the AOM and only $0.78 \%$ lower than the value of the SM. It can be observed that both of the two proposed methods are superior to the empirical operations in actual operations, and the performance of the DM is a little inferior to the SM. This result is quite suitable and meaningful for the actual operations.

\begin{tabular}{|c|c|c|c|}
\hline Planning method & DM & SM & AOM \\
\hline $\begin{array}{l}\text { Average objective value } \\
\text { Average solution time (s) } \\
\text { Gap (\%) } \\
\text { Difference in the total cost between }\end{array}$ & $\begin{array}{c}643167.5 \\
834.44 \\
34.8 \%\end{array}$ & $\begin{array}{c}638087.2 \\
314.38 \\
35.4 \%\end{array}$ & $\begin{array}{l}987950.1^{*} \\
\text { N/A } \\
0.0\end{array}$ \\
\hline
\end{tabular}

Table 1. Comparison of different methods

It is worth mentioning that out-of-stock situation occurred during the AOM test (we use the symbol $*$ to label it). A shortage of the third kind of medical resources was appeared at the $7^{\text {th }}$ week and the $25^{\text {th }}$ week, with a quantity of 3 and 6 , respectively. However, this phenomenon does not occur in both the DM and the SM. The reason is that both the order time and order quantity in these two models are decision variables and would be systematically optimized, 
while both the order time and order quantity in AOM are pre-set. As introduced in Section 1, if the important parameters, such as order/shipment frequency and order quantity, are manually determined by the decision maker based on his/her experience, the planned schedules may not be efficient, or may not be at all feasible to satisfy all demands since a large portion of customer service requests in CHSCs are uncertain and tine-varying.

\subsection{Sensitivity Analysis}

To understand the influence of stochastic demand on the solution, we perform sensitivity analysis of the change of demand to the operation cost. The proposed models in Section 3 provide several key parameters that may affect the final result, i.e., the average demand of medical resources in each planning week, the standard deviation setting, and the capacity of DCDC and CHSC, etc. The sensitivity analyses of these parameters are shown as follows.

To detect the influence of the average demand on the final solution, the value of it is adjusted with four different values $(-20 \%,-10 \%, 10 \%$ and $20 \%)$. The results are shown in Table 2 . The total operation cost is increasing along with the growth of the average demand, regardless of which planning method is used as a basis (from $-24.00 \%$ to $24.41 \%$, from $-24.58 \%$ to $24.67 \%$, and from $-13.73 \%$ to $10.79 \%$, respectively). This suggests us that the higher the average demand of medical resources, the higher the operation cost. If the decision makers can find a way to reduce the average demand, i.e., informing people to prevent the epidemic by using internet, radio and television, and thus reduce the actual demand of medical resources, the total operation cost can be reduced.

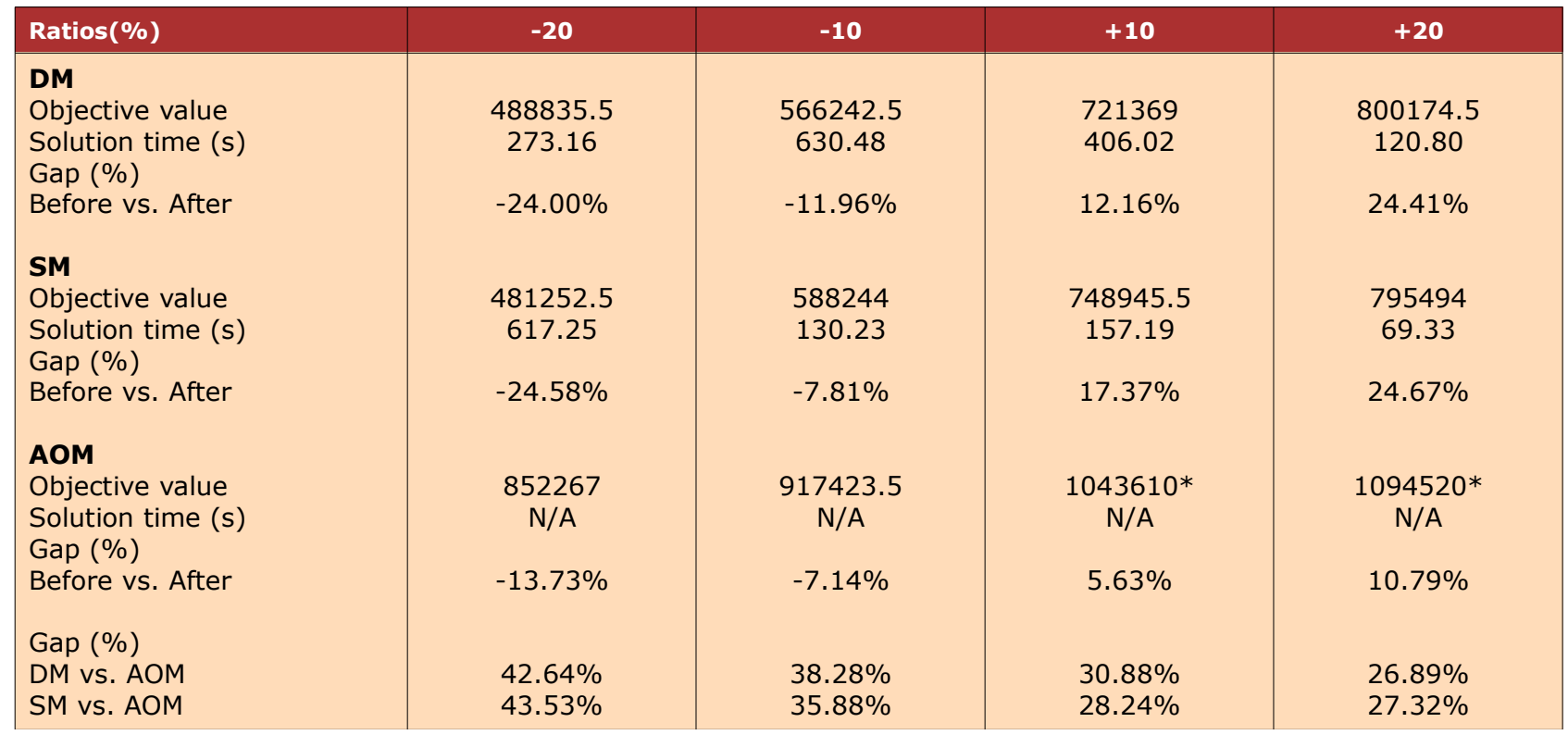

Table 2. Sensitivity analysis with the change of average demand 
It can also be observed that the difference between the DM and the SM is negligible, no matter what the average demand is. However, difference between the DM and the AOM decreases from $42.64 \%$ to $26.89 \%$, and it varies from $43.53 \%$ to $27.32 \%$ when it is compared between the SM and the AOM. This result suggests that the proposed two methods produce better planned results, especially when the average demand is lower.

\begin{tabular}{|c|c|c|c|c|}
\hline Value & 8 & 9 & 11 & 12 \\
\hline $\begin{array}{l}\text { SM } \\
\text { Objective value } \\
\text { Solution time (s) } \\
\text { Gap (\%) } \\
\text { Before vs. After } \\
\text { AOM } \\
\text { Objective value } \\
\text { Solution time (s) } \\
\text { Gap (\%) } \\
\text { Before vs. After } \\
\text { Gap (\%) } \\
\text { Difference in the total cost } \\
\text { between SM and AOM }\end{array}$ & 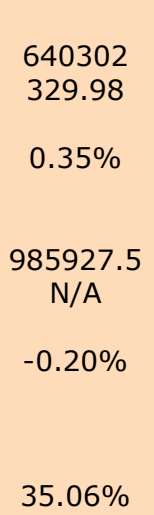 & $\begin{array}{c}644415 \\
535.19 \\
0.99 \% \\
985668 \\
\text { N/A } \\
-0.23 \%\end{array}$ & $\begin{array}{c}657644.5 \\
720.34 \\
3.06 \% \\
\\
993108 \\
\text { N/A } \\
0.52 \%\end{array}$ & $\begin{array}{c}668352 \\
987.69 \\
4.74 \% \\
\begin{array}{c}1001154 * \\
\text { N/A }\end{array} \\
1.34 \%\end{array}$ \\
\hline
\end{tabular}

Table 3. Sensitivity analysis of the standard deviation

To investigate the influence of standard deviation on the final solution, we test four values of the standard deviation (8, 9, 11 and 12). As the standard deviation increases, the stochastic demand can be generated in a larger range. The results are shown in Table 3. The operation cost is increasing along with the standard deviation, whatever in SM or AOM. The difference in the operation cost between the SM and the AOM decreases from $35.06 \%$ to $33.24 \%$ as the standard deviation increases. Although there are only small differences among these objective values (from $0.35 \%$ to $4.74 \%$ and from $-0.20 \%$ to $1.34 \%$, respectively), more time is required to solve the problem as the standard deviation increases (from $329.98 \mathrm{sec}$ to $987.69 \mathrm{sec}$ in $\mathrm{SM})$. This suggests that the more stable of the demand, the lower of the operation cost and the better of the solution performance.

To investigate the influence of the capacity of the organizations on the performances of the three different methods, we test four values of the parameters. As shown in Table 4, the operation cost decreases about $0.3 \%$ when the capacity of DCDC and CHSCs respectively increases $10 \%$, whatever in DM or SM. It can also be found that only small differences among these objective values. However, $5 \%$ of the operation cost is increased when the capacity of DCDC and CHSC is respectively increased $10 \%$ in the AOM. Moreover, difference between the DM and the AOM is increased from $39.01 \%$ to $47.00 \%$ as the value of ratio increases. Similarly, difference between the SM and the AOM is varied from $38.91 \%$ to $46.91 \%$. This suggests us that the capacity of the medical institutions can strongly influence the total 
operation cost in our actual operations. However, when the proposed two methods are applied, such influence can be decreased greatly.

\begin{tabular}{|c|c|c|c|c|}
\hline Ratios(\%) & +10 & +20 & +30 & +40 \\
\hline $\begin{array}{l}\text { DM } \\
\text { Objective value } \\
\text { Solution time (s) } \\
\text { Gap (\%) } \\
\text { Before vs. After } \\
\text { sM } \\
\text { Objective value } \\
\text { Solution time (s) } \\
\text { Gap (\%) } \\
\text { Before vs. After } \\
\text { AOM } \\
\text { Objective value } \\
\text { Solution time (s) } \\
\text { Gap (\%) } \\
\text { Before vs. After } \\
\text { Gap (\%) } \\
\text { DM vs. AOM } \\
\text { SM vs. AOM }\end{array}$ & $\begin{array}{c}640790.5 \\
110.81 \\
-0.37 \% \\
\\
641843 \\
1034.36 \\
0.59 \% \\
1050569 \\
\text { N/A } \\
6.34 \%\end{array}$ & $\begin{array}{l}639078 \\
484.59 \\
-0.64 \% \\
639906 \\
524.64 \\
0.29 \%\end{array}$ & $\begin{array}{c}1149399 \\
\text { N/A } \\
16.34 \% \\
\\
44.55 \% \\
44.48 \%\end{array}$ & $\begin{array}{c}1198814 \\
\text { N/A } \\
21.34 \% \\
\\
47.00 \% \\
46.91 \%\end{array}$ \\
\hline
\end{tabular}

Table 4 . Sensitivity analysis of the change ratio of capacity

\section{Conclusion}

In this study, a time-space network technique is applied to formulate the medical resources order and shipment scheduling in community health service centers. A deterministic planning model is presented to depict medical resources order and shipment with a pre-ascertained demand. A stochastic planning model is then developed to respond to the uncertain demand. A solution procedure is developed to efficiently solve the proposed models and a simulationbased evaluation method is also developed to compare the performances of the models. Numerical tests, relating to some health service departments' operations, are performed to evaluate the proposed models and the actual operations. The main contributions of this work to the literature are as follows:

(1) While most research on medical resources optimization studies a static problem taking no consideration of the time evolution and especially the dynamic demand for such resources (Sun et al., 2014; Liu \& Liang, 2013; Liu \& Zhao, 2012), the proposed models in our work integrate time-space network technique, which can find the optimal scheduling of logistical support for medical resources order and shipment in CHSCs effectively.

(2) The logistics plans in response to the deterministic demand and the time-varying demand are constructed as a 0-1 mixed integer programming model and a stochastic 
integer programming model, respectively. The optimal solutions not only minimize the operation cost of the logistics system, but also can improve the order and shipment operation in practice.

Future research would be useful in the following directions. Initially, although it is reasonable to assume that the government can ensure the adequate supply of the needed medical resources, out-of-stock situation could be a meaningful topic of future research. Secondly, we did not consider shipment routing in this work. Actually, it would be more useful in application if the model considers these two aspects. Certainly, the development of other models using other methods for solving this type of problem and comparing the results with those of our model could also be a direction of future research.

\section{Acknowledgement}

This work was supported by the National Natural Science Foundation of China under Grant (No.71301076, 71401075); Natural Science Foundation of Jiangsu Province under Grant (BK20130771) and the Research Fund for the Doctoral Program of Higher Education of China under Grant (20133219120037). The authors also gratefully acknowledge the Zijin Intelligent Program under Grant (No. 2013-ZJ0211) in Nanjing University of Science and Technology.

\section{References}

Beraldi, P., Bruni, M.E., \& Conforti, D. (2004). Designing robust emergency medical service via stochastic programming. European Journal of Operational Research, 158, 183-193. http://dx.doi.org/10.1016/S0377-2217(03)00351-5

Dasaklis, T.K., Pappis, C.P., \& Rachaniotis, N.P. (2012). Epidemics control and logistics operations: A review. International Journal of Production Economics, 139, 393-410. http://dx.doi.org/10.1016/j.ijpe.2012.05.023

Dib, H.H., Sun, P., Zhu, M.M., Wei, S.H., \& Li, L. (2010). Evaluating community health centers in the City of Dalian, China: How satisfied are patients with the medical services provided and their health professionals? Health \& Place, 16, 477-488. http://dx.doi.org/10.1016/j.healthplace.2009.12.005

Duintjer Tebbens, R.J., Pallansch, M.A., Alexander, J.P., \& Thompson, K.M. (2010). Optimal vaccine stockpile design for an eradicated disease: Application to polio. Vaccine, 28, 4312-4327. http://dx.doi.org/10.1016/j.vaccine.2010.04.001 
Holte, M., \& Mannino, C. (2013). The implementor/adversary algorithm for the cyclic and robust scheduling problem in health-care. European Journal of Operational Research, 226, 551-559. http://dx.doi.org/10.1016/j.ejor.2012.10.029

Kliewer, N., Mellouli, T., \& Suhl, L. (2006). A time-space network based exact optimization model for multi-depot bus scheduling. European Journal of Operational Research, 175, 1616-1627. http://dx.doi.org/10.1016/j.ejor.2005.02.030

Li, H., \& Yu, W. (2011). Enhancing community system in China's recent health reform: An effort to improve equity in essential health care. Health Policy, 99, 167-173. http://dx.doi.org/10.1016/j.healthpol.2010.08.006

Liu, M., \& Liang, J. (2013). Dynamic optimization model for allocating medical resources in epidemic controlling. Journal of Industrial Engineering and Management, 6, 73-88. http://dx.doi.org/10.3926/jiem.663

Liu, M., \& Zhao, L.D. (2012). An integrated and dynamic optimization model for the multi-level emergency logistics network in anti-bioterrorism system. International Journal of Systems Science, 43, 1464-1478. http://dx.doi.org/10.1080/00207721.2010.547629

Nikakhtar, A., \& Hsiang, S.M. (2014). Incorporating the dynamics of epidemics in simulation models of healthcare systems. Simulation Modelling Practice and Theory, 43, 67-78. http://dx.doi.org/10.1016/j.simpat.2014.01.007

Rachaniotis, N.P., Dasaklis, T.K., \& Pappis, C.P. (2012). A deterministic resources scheduling model in epidemic control: A case study. European Journal of Operational Research, 216, 225-231. http://dx.doi.org/10.1016/j.ejor.2011.07.009

Rottkemper, B., Fischer, K., \& Blecken, A. (2012). A transshipment model for distribution and inventory relocation under uncertainty in humanitarian operations. Socio-Economic Planning Sciences, 46, 98-109. http://dx.doi.org/10.1016/j.seps.2011.09.003

Savachkin, A., \& Uribe, A. (2012). Dynamic redistribution of mitigation resourcess during influenza pandemics. Socio-Economic Planning Sciences, 46, 33-45. http://dx.doi.org/10.1016/j.seps.2011.05.001

Sun, L., DePuy, G.W., \& Evans, G.W. (2014). Multi-objective optimization models for patient allocation during a pandemic influenza outbreak. Computers \& Operations Research, 51, 350-359. http://dx.doi.org/10.1016/j.cor.2013.12.001

Vlah, J.S., \& Rui, F.J. (2012). Multi-objective scheduling and a resources allocation problem in hospitals. Journal of Scheduling, 15, 513-535. http://dx.doi.org/10.1007/s10951-012-0278-9 
Xu, M., Wong, T.C., \& Chin, K.S. (2014). A medical procedure-based patient grouping method for an emergency department. Applied Soft Computing, 14, 31-37. http://dx.doi.org/10.1016/j.asoc.2013.09.022

Yan, S.Y, Lin, C.K., \& Chen, S.Y. (2014). Logistical support scheduling under stochastic travel times given an emergency repair work schedule. Computers \& Industrial Engineering, 67, 20-35. http://dx.doi.org/10.1016/j.cie.2013.10.007

Yan, S.Y., Lin, J.R., \& Lai, C.W. (2013). The planning and real-time adjustment of courier routing and scheduling under stochastic travel times and demands. Transportation Research Part E: Logistics and Transportation Review, 53, 34-48. http://dx.doi.org/10.1016/j.tre.2013.01.011

Yan, S.Y., Shih, Y.L., \& Shiao, F.Y. (2008). Optimal cargo container loading plans under stochastic demands for air express carriers. Transportation Research Part E: Logistics and Transportation Review, 44, 555-575. http://dx.doi.org/10.1016/j.tre.2007.01.006

Yan, S.Y., Tang, C.H., \& Fu, T.C. (2008). An airline scheduling model and solution algorithms under stochastic demands. European Journal of Operational Research, 190, 22-39. http://dx.doi.org/10.1016/j.ejor.2007.05.053

Zhang, Z.H., \& Jiang, H. (2014). A robust counterpart approach to the bi-objective emergency medical service design problem. Applied Mathematical Modelling, 38, 1033-1040. http://dx.doi.org/10.1016/j.apm.2013.07.028

Journal of Industrial Engineering and Management, 2015 (www.jiem.org)

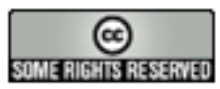

Article's contents are provided on an Attribution-Non Commercial 3.0 Creative commons license. Readers are allowed to copy, distribute and communicate article's contents, provided the author's and Journal of Industrial Engineering and Management's names are included. It must not be used for commercial purposes. To see the complete license contents, please visit http://creativecommons.org/licenses/by-nc/3.0/. 